\title{
Lung Perfusion SPECT: Application in a Patient With Tetralogy of Fallot and Suspected Pulmonary Thromboemboli
}

\author{
Mina Ranji Amjad ${ }^{1}$; Mehrshad Abbasi ${ }^{2}$; Saeed Farzanehfar ${ }^{2, *}$ \\ ${ }_{1}^{1}$ Research Institute for Nuclear Medicine, Shariati Hospital, Tehran University of Medical Sciences, Tehran, Iran \\ ${ }^{2}$ Department of Nuclear Medicine, Vali-Asr Hospital, Tehran University of Medical Sciences, Tehran, Iran \\ *Corresponding author: Saeed Farzanehfar, Department of Nuclear Medicine, Vali-Asr Hospital, Tehran University of Medical Sciences, P. O. Box: 14197-33141, Tehran, Iran. \\ Tel:+98-2161192400, Fax: +98-2166581529, E-mail: farzanehfar@tums.ac.ir
}

Received: November 11, 2012; Revised: March 4, 2013; Accepted: April 8, 2013

\begin{abstract}
A 22-year-old woman presented with acute left-sided pleuritic chest pain and dyspnea 6 days after surgery for revision of the stenotic central aortopulmonary shunt. She had a history of tetralogy of Fallot(TOF), pulmonary valve stenosis, ventricular septal defect and major aortopulmonary collateral artery. Her Waterston shunt was placed when she was 5 years old and stented and re-dilated after stenosis. Acute pulmonary thromboemboli (PTE) was suspected and pulmonary perfusion scan was performed with $4 \mathrm{mCi} 99 \mathrm{~m}$ Technetium labeled macroaggregated albumin. The left lung was globally hypoperfused with evident uptake in the brain, renal parenchyma and thyroid. SPECT images revealed a segmental wedge-shaped peripheral defect in the posterior segment of the left upper lobe. The scan was interpreted as acute/chronic PTE or vascular abnormality. CT angiography excluded PTE; nevertheless the patient was treated with a therapeutic dose of heparin changed to warfarin and was discharged with improvement of the symptoms. Pulmonary artery angiography was not performed.
\end{abstract}

Keywords: Tomography, Emission-Computed, Single-Photon; Tetralogy of Fallot; Pulmonary Embolism

\section{Introduction}

The SPECT acquisition in lung perfusion scan provides higher sensitivity and specificity to detect pulmonary thromboemboli (PTE) compared to planar imaging (1, $2)$. Because of higher specificity, there are hopes that the lung perfusion SPECT could be interpreted without a ventilation study. The value of higher specificity is more highlighted in complicated cases with vascular abnormality and shunts. In this report, a case with tetralogy of Fallot (TF) and multiple cardiovascular abnormalities and corrective shunts are presented underscoring the importance of SPECT imaging in complicated patients.

\section{Case Presentation}

A 22-year-old female patient was admitted for surgery to revise her Waterston central aortopulmonary shunt. She had the history of TF, pulmonary valve stenosis, ventricular septal defect and major aortopulmonary collateral artery. Her shunt was placed at the age of 5 years then complicated with stenosis for which a stent was placed and re-dilated 1 year ago. After echocardiography reported minimal aorto-pulmonary shunt flow, a decision was made to operate the patient for revision of the central shunt. Midsternal incision was done and a polytetrafluoroethylene (PTFE) tube was inserted between the ascending aorta and right pulmonary branch, where the previous Gortex was located. Patient recovery and rehabilitation after surgery was optimal until the patient experienced abrupt left-sided pleuritic chest pain and dyspnea. Arterial $\mathrm{O}_{2}$ saturation diminished to $75 \%$. The patient was referred to the department of nuclear medicine for perfusion and ventilation lung scanning. Perfusion lung scan was done with $4 \mathrm{mCi}$ 99m Technetium labeled macroaggregated albumin (total particle count 120,000) in planar anterior, posterior, lateral and multiple oblique projections (Figure 1). The left lung was generally hypoperfused more in the upper zone. No clear evidence of PTE was detected in the planar views. Additionally, the brain, renal parenchyma and the thyroid demonstrated uptake due to the right to left shunt (Figure 2). Ventilation scan was not performed due to logistic problems; the available ventilation tracer in Iran is $81 \mathrm{Kr}$, which is distributed only on Wednesdays. Afterward, a 2-hour delayed SPECT imaging was done (Figure 3). There was a large wedge-shaped peripheral defect in the apicoposterior segment of the left upper lobe. The finding was interpreted as either acute/chronic PTE or secondary to developmental/iatrogenic vascular abnormality. Therapeutic dose of intravenous IV heparin was administrated (500 U/hour). Meanwhile, the patient underwent CT angiography reporting postoperative atelectatic changes in the bilateral lower lobes without evidence in support of PTE. The IV heparin was changed

Copyright ( ) 2015, Tehran University of Medical Sciences and Iranian Society of Radiology. This is an open-access article distributed under the terms of the Creative Commons Attribution-NonCommercial 4.0 International License (http://creativecommons.org/licenses/by-nc/4.0/) which permits copy and redistribute the material just in noncommercial usages, provided the original work is properly cited. 


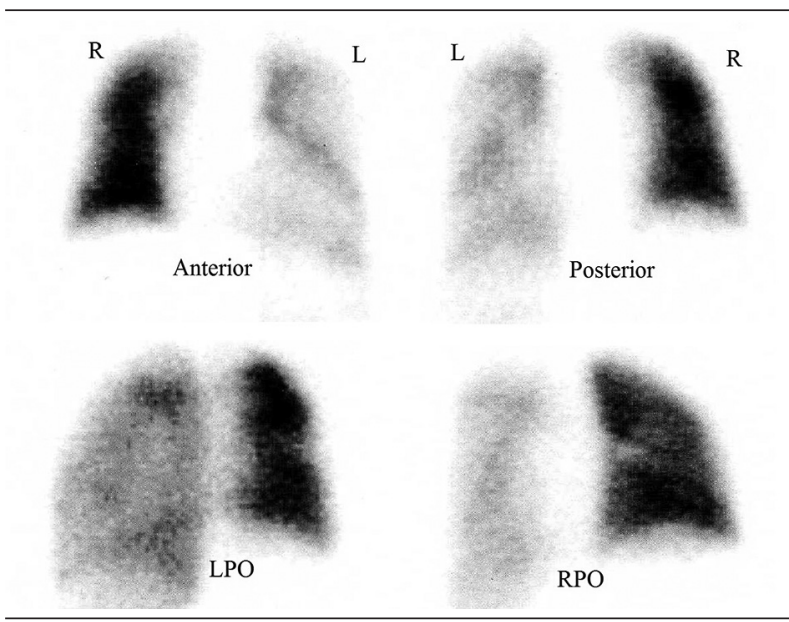

Figure 1. Multiple perfusion scan projections of a 22-year-old patient with history of tetralogy of Fallot and revision of Waterston shunt presented with post operation dyspnea. The left lung is globally hypoperfused more in the upper zone without clear evidence indicative of pulmonary thromboemboli

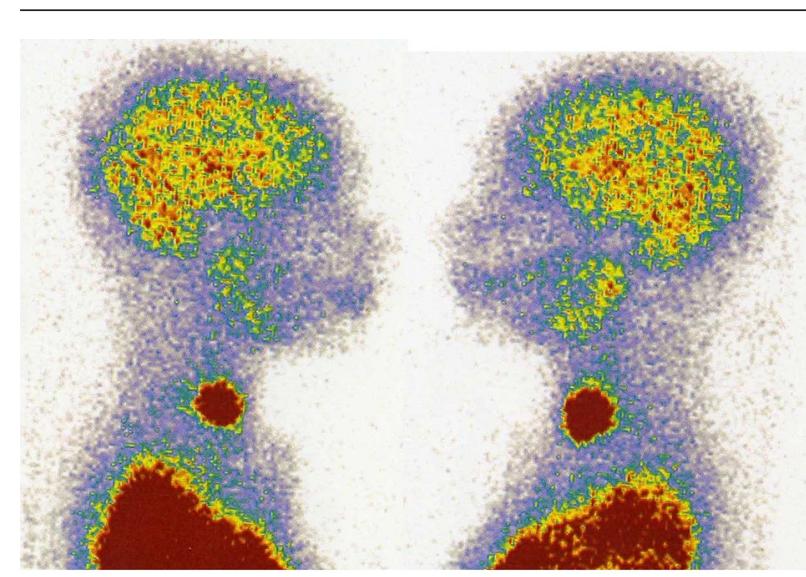

Right Lateral

Left Lateral

Figure 2. Abnormal uptake of $99 \mathrm{mTc}$ macroaggregated albumin in the brain and thyroid indicative of right to left shunt

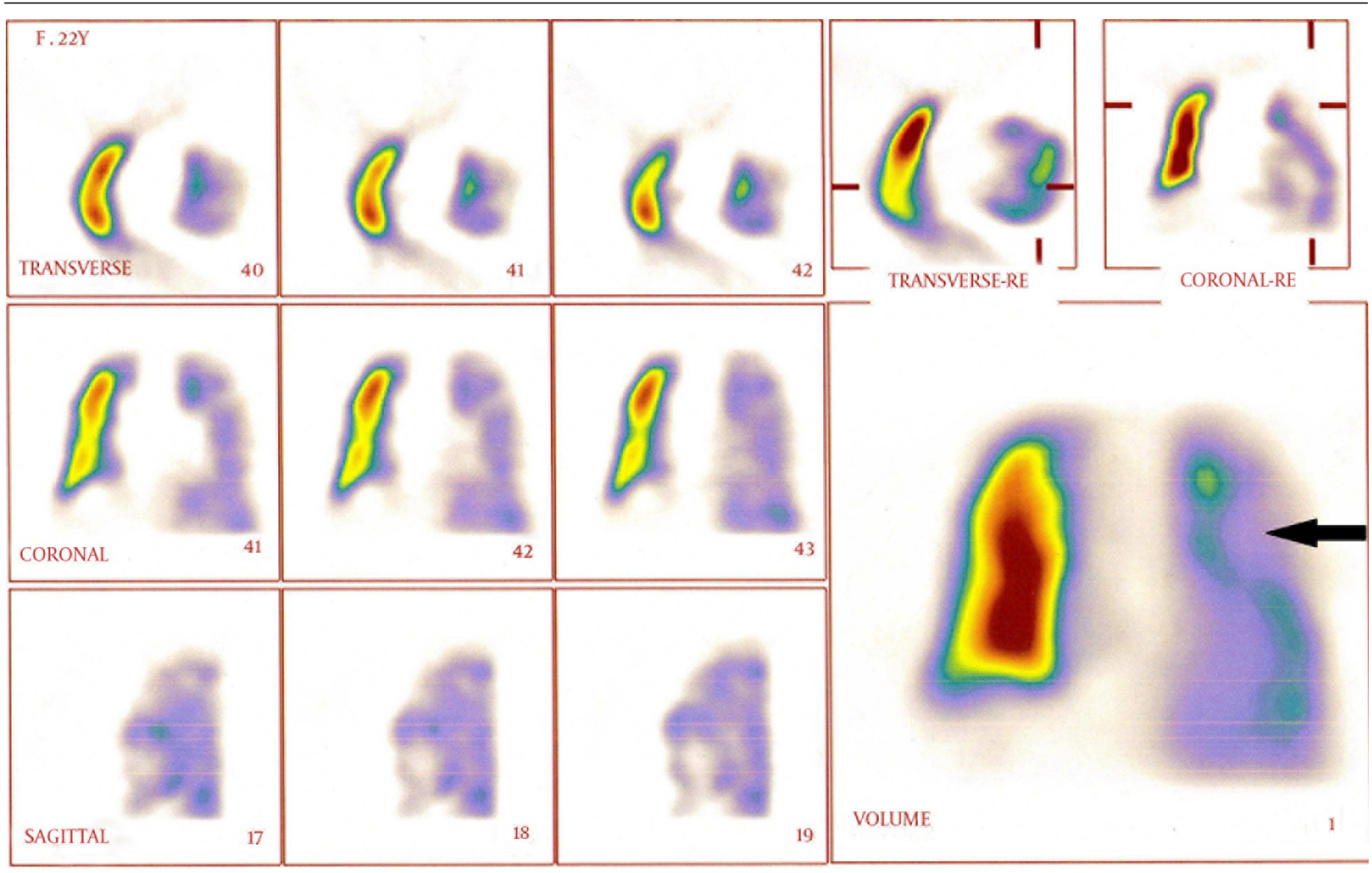

Figure 3. Lung perfusion SPECT images illustrating a segmental wedge shaped peripheral defect in the apicoposterior segment of the upper lobe of the globally hypoperfused left lung (arrow)

to warfarin after 5 days when the symptoms improved gradually and the arterial $\mathrm{O} 2$ saturation increased to $92 \%$ in room atmosphere. Pulmonary angiography was not performed as the patient was improving. She was discharged with good general condition and no specific pulmonary symptoms.

\section{Discussion}

Tetralogy of Fallot is a congenital cyanotic heart disease in about $3.5 \%$ of infants with congenital heart diseases. There are a wide spectrum of anatomic defects with varying severity including ventricular septal defect, overriding aorta, right ventricular outflow tract 
obstruction, and right ventricular hypertrophy (3). Congenital heart disease is a risk factor for pulmonary emboli. Diagnosis of pulmonary emboli is a great diagnostic challenge and it should be suspected in any patient with unexplained dyspnea, tachypnea, or chest pain $(4,5)$.

Lung perfusion and ventilation SPECT is superior to planar scan in detecting pulmonary embolism with a high sensitivity and specificity (6). Perfusion lung scan without ventilation scan has also been shown to have a high specificity of $92 \%$ and sensitivity of $87 \%$ (7). The advantages of lung perfusion SPECT imaging include higher contrast for better detection of the shape and location of defects and higher sensitivity (6). In complicated cases similar to the patient described here, this is of foremost importance. Because pulmonary angiography was not performed and CT angiography was negative, PTE was not confirmed in this case. Hence, the patient improved with therapy. This may foster the diagnosis of PTE. CT angiography is a sensitive and optimal technique for diagnosing large pulmonary emboli, but it has a relatively low sensitivity (below $80 \%$ ) for subsegmental PTEs (4).

\section{Authors' Contributions}

Mina Ranji Amjad and Saeed Farzanehfar introduced the case and followed the outcome and wrote the manuscript with cooperation of Mehrshad Abbasi.

\section{References}

1. Gutte H, Mortensen J, Jensen CV, von der Recke P, Petersen CL, Kristoffersen US, et al. Comparison of V/Q SPECT and planar V/Q lung scintigraphy in diagnosing acute pulmonary embolism. Nucl Med Commun. 2010;31(1):82-6.

2. Collart JP, Roelants V, Vanpee D, Lacrosse M, Trigaux JP, Delaunois $\mathrm{L}$, et al. Is a lung perfusion scan obtained by using single photon emission computed tomography able to improve the radionuclide diagnosis of pulmonary embolism? Nucl Med Commun. 2002;23(11):1107-13.

3. Apitz C, Webb GD, Redington AN. Tetralogy of Fallot. Lancet. 2009;374(9699):1462-71.

4. Langan CJ, Weingart S. New diagnostic and treatment modalities for pulmonary embolism: one path through the confusion. $M t$ Sinai JMed. 2006;73(2):528-41.

5. Healy F, Hanna BD, Zinman R. Pulmonary complications of congenital heart disease. Paediatr Respir Rev. 2012;13(1):10-5.

6. Laurence IJ, Redman SL, Corrigan AJ, Graham RN. V/Q SPECT imaging of acute pulmonary embolus - a practical perspective. Clin Radiol. 2012;67(10):941-8.

7. Marini C, Palla A, Giuntini C. Pulmonary embolism: lung scan and computed tomography. Ital Heart J. 2005;6(10):811-7. 